\title{
Primary wound closure with a Limberg flap vs. secondary wound healing after excision of a pilonidal sinus: a multicentre randomised controlled study
}

\author{
S. A. Käser • R. Zengaffinen • M. Uhlmann • \\ C. Glaser • C. A. Maurer
}

Accepted: 25 October 2014 / Published online: 5 November 2014

(C) Springer-Verlag Berlin Heidelberg 2014

\begin{abstract}
Purpose Off-midline procedures seem to be the best method of primary wound closure after excision of a pilonidal sinus. Primary wound closure with a Limberg flap was compared to secondary wound healing.

Methods From January 2006 to July 2012, 102 patients with given informed consent (mean age 28 years, men $81 \%$ ) who had excision of a pilonidal sinus in three hospitals in Switzerland were randomised to group L (Limberg flap, $n=51$ ) or to group $\mathrm{E}$ (excision only, $n=51$ ). Primary endpoint was duration of incapacity for work. Follow-up was at 3 weeks and at 1 year postoperative ( $95 \%$ follow-up).

Results Both groups were comparable with regard to patient characteristics. The median (range) operation time was 60 $(30-80) \mathrm{min}$ in group L vs. $30(10-75) \mathrm{min}$ in group E $(p<0.001)$. No significant differences were found in postoperative pain and painkiller intake; pain, percentage of patients at work and overall satisfaction at 3 weeks postoperative; and overall duration of incapacity for work and overall satisfaction at 1 -year follow-up. The complication rate was $49 \%$ in group $\mathrm{L}$ vs. $12 \%$ in group $\mathrm{E}(p<0.001)$. Complications in group $\mathrm{L}$ were seroma (6\%), wound dehiscence ( $45 \%$ ), skin necrosis (10\%), hematoma (6\%), infection ( $4 \%$ ) and recurrent disease
\end{abstract}

S. A. Käser · M. Uhlmann · C. Glaser · C. A. Maurer $(\bowtie)$ Department of General, Visceral, Vascular, and Thoracic Surgery, Hospital of Baselland, affiliated to the University of Basel, Rheinstrasse 26, 4410 Liestal, Switzerland

e-mail: christoph.maurer@hin.ch

S. A. Käser · R. Zengaffinen

Department of General, Visceral and Trauma Surgery, Hospital Centre of Oberwallis (SZO), Pflanzettastrasse 6, 3930 Visp, Switzerland

C. Glaser

Department of General, Visceral and Trauma Surgery, Hospital Centre Fricktal, Riburgerstrasse 12, 4310 Rheinfelden, Switzerland
$(13 \%)$. Complications in group E were recurrent disease $(6 \%)$ and wound healing disorder $(6 \%)$.

Conclusions After excision of a pilonidal sinus, primary wound closure with a Limberg flap has no advantage over secondary wound healing. The main reason for this conclusion is the relatively high complication rate of primary wound closure with a Limberg flap.

Keywords Pilonidal sinus disease $\cdot$ Limberg flap $\cdot$ Excision . Primary wound closure $\cdot$ Secondary wound healing

\section{Introduction}

The incidence of pilonidal sinus disease has risen in the past decades [1], and the currently estimated incidence in the general population is 26 per 100,000 per year $[2,3]$. The prevalence of pilonidal sinus disease can be up to 88 per 1000 in high-risk groups such as in young Turkish soldiers [4]. The socioeconomic burden of pilonidal sinus disease is high as, first, mainly young people between 20 and 30 years of age are at risk [5]; second, treatment takes weeks to months [2]; and third, reported overall recurrence rates at 20 years follow-up reach $34 \%$ [6].

The optimal therapy for pilonidal sinus disease is still under debate [2], and a wide variation of different surgical techniques is still in use [7]. No clear benefit has been shown for secondary wound healing compared to surgical closure [2]. If surgical closure is chosen, off-midline closure with flap procedures, such as the Limberg flap procedure $[8,9]$, or the Karydakis procedure [10], is recommended [2, 11-13]. Although one randomised controlled study with $n=49$ patients comparing the Limberg flap procedure to secondary wound healing showed results in favour of the Limberg flap procedure [14], another randomised study with $n=140$ patients comparing the Limberg flap procedure to unroofing and 
marsupialisation showed results rather in favour of secondary wound healing [15]. Thus, there is not enough evidence to recommend the Limberg flap procedure instead of secondary wound healing for pilonidal sinus disease [2].

For these reasons, we aimed to compare primary wound closure with a Limberg flap to secondary wound healing without vacuum wound dressing after excision of a pilonidal sinus.

\section{Patients and methods}

Ethics and insurance

The regional ethics committees (Ethics Committee of Basel (EKBB Nr. 234/05), Aargau and Valais) approved this multicentre randomised controlled study. All patients were insured for adverse outcomes (Axa-Winterthur Versicherung AG, policy number 14.024.750/ZNW). Further, the study was registered at clinicaltrials.gov (NCT 01889394).

\section{Study endpoints}

The primary endpoint was the duration of incapacity for work. Secondary endpoints were perioperative pain, pain at 3 weeks postoperative, complication rate, recurrence rate at 1 year and patient satisfaction at 1 year.

\section{Biometrics and statistics}

$H_{A}$ hypothesis A difference between the duration of incapacity for work is expected between the patients with the Limberg flap procedure and those with excision only as treatment for pilonidal sinus disease.

The biometry was done with the assumption that the rate of incapacity for work after 3 weeks would be $50 \%$ for the group with the Limberg flap, compared to $65 \%$ for excision only. This assumption was based on the varying reported median duration of incapacity for work between 1 and 5 weeks postoperatively $[16,17]$. The type I and II error was 0.05 and 0.20 , respectively. The estimated sample size was $n=100$. In order to compensate for study dropouts, the chosen final sample size was $n=102$.

Results were expressed as mean and standard deviation or median and range. For statistical computing, Stata 10.0 for Windows was used. For statistical analysis, the two-sided Fisher's exact test was used for categorical data and the Wilcoxon rank-sum test was used for numerical data. $p$ values below 0.05 were considered to be significant.

\section{Randomisation}

In the main participating centre, a randomisation list was created by a computer-based method with the same number of patients in both groups. From this list, sealed nontransparent envelopes were created and numbered sequentially. The randomisation list was closed away.

The randomisation in the second participating centre was done by phone call to the main centre with the same randomisation envelopes. The third participating centre, joining the study in the year 2009, had a lucky dip system with 15 tickets for the Limberg flap procedure and 15 tickets for the excision only procedure.

Patient recruitment and inclusion/exclusion criteria

Oral and written informed consent was obtained from each patient willing to participate in this study. To permit primary wound closure and to prevent recurrences, apparent abscesses were treated with incision and antibiotic therapy for intended 12 to 14 days (median of 13 days) until the elective definitive surgical treatment was done [6]. If the general practitioner had already done a relieving incision before the first presentation in hospital, the interval to definitive surgical treatment was assessed as well.

Just prior to scheduled elective surgery, the responsible surgeon checked the inclusion and exclusion criteria. The only inclusion criterion was the unequivocal finding of pilonidal sinus disease. Exclusion criteria were age $<18$ years, lack of informed consent, florid abscess at the time of the elective surgery, pregnancy, immunosuppression, dermatological diseases and prior local flap procedures.

Eligible patients with written informed consent were then randomised.

\section{Surgical technique}

Patients were positioned in the prone jack-knife position, and the buttocks were drawn to the side by an adhesive tape. Single-shot antibiotic prophylaxis with cefuroxime $1.5 \mathrm{~g}$ was administered intravenously, although this step is debatable [18]. The surgery was planned by doing a draft of the excision ( \pm Limberg flap) on the skin. The pores were filled with methylene blue and all the coloured tissue was completely removed, suggesting radical excision of the pilonidal sinus.

In the case of randomisation for excision only, the shape of the resulting cavity was only adapted to the extent of the disease; however, the excision was done down to the presacral fascia. After haemostasis, the wound was covered with sterile gauze.

In the case of randomisation for the Limberg flap procedure, a rhomboid excision was done, followed by primary wound closure with the classical Limberg flap procedure as demonstrated in Fig. 1. Some surgeons tailored a round edge at the tip of the Limberg flap, while others tailored a sharp edge as shown in Fig. 1. 


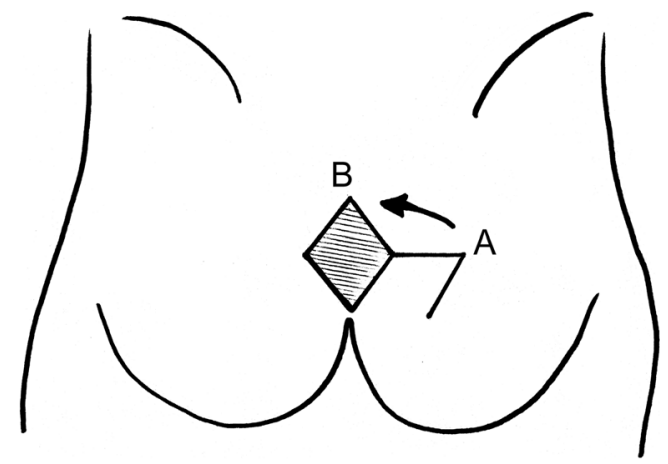

Fig. 1 Principle of the Limberg flap; the tip of the flap "A" is transposed to point "B"

The flap included the skin and the subcutaneous tissue without the fascia of the gluteus muscle. The subcutaneous tissue was sutured with absorbable polyfil sutures (polyglactine) and the skin was sutured with monofil nonabsorbable sutures (polypropylene or nylon). It was up to the responsible surgeon to decide the necessity of wound drainage (drainage rate $49 \%$ ) despite lack of evidence [19]. Patients with the Limberg flap procedure had prolonged antibiotic therapy prescribed postoperatively (amoxicillin and clavulanic acid $2 \times 1 \mathrm{~g}$ per day or cefuroxime $500 \mathrm{mg} 2 \times 1 \mathrm{~g}$ per day for 7 days postoperative) [20].

Each specimen was sent to the pathology department for histological examination.

All patients were kept in the hospital for at least $24 \mathrm{~h}$. Before discharge, every patient had instructions about optimal wound care by the surgeons and by the nurse staff. If necessary, home visiting nurse services provided optimal wound care, and all patients had regular visits at their general practitioner. For optimal treatment, patients with wound breakdown after the Limberg flap procedure or wound healing disorder after the excision only procedure had regular visits in the consultation hours of the attending surgeons in hospital.

\section{Assessment of baseline data}

Before surgery, presence of recurrence, localization and number of pores and the distance between the most caudal porus and the anus were assessed. Questionable risk factors for unfavourable postoperative outcome such as obesity and smoking with lack of evidence were not assessed [21].

During surgery, presence of pus, the dimensions, and the volume of the cavity after excision were assessed. The volume of the cavity was measured by filling it with sterile saline solution. After surgery, presence of wound tension, presence of drainage and the operation time were assessed. The pathology reports were analysed for the dimensions of the specimen, the completeness of resection, the presence of abscess and of granulocytes, inclusion of hair and the confirmation of diagnosis.

\section{Assessment of outcomes}

At discharge, the pain score and the need for painkiller medication were assessed.

The patient's general practitioner defined the length of the incapacity for work. Students or unemployed patients defined the time point when they were able to do their usual daily activities themselves.

The first follow-up at 3 weeks postoperative by an attending surgeon was done by interview and clinical examination using a standardised form. The following factors were assessed at 3 weeks: duration of incapacity for work, pain intensity on a graduated scale from 0 to 10 (no pain to worst pain), complications (wound dehiscence, infection, necrosis, hematoma, other) and patient satisfaction on a graduated scale from 0 to 10 (not satisfied at all to completely satisfied).

The second follow-up at 1 year postoperative was done by phone call by the study doctor and not by the attending surgeon who did the operation.

The following factors were assessed at 1 year: duration of incapacity for work, complications, recurrent disease and patient satisfaction on a graduated scale from 0 to 10 (not satisfied at all to completely satisfied). Recurrent disease was defined as necessity for re-operation for pilonidal sinus disease and/or presence of oozing in the sacrococcygeal region. In cases of ambiguity, the patient was seen in the consultation hour.

This study adheres to the Consort 2010 checklist of information to include when reporting a randomised trial [22].

\section{Results}

Between January 2006 to July 2012, $n=105$ patients gave their informed consent and were recruited for the study in three hospitals in Switzerland. One patient refused participation after giving informed consent, one patient had a dermatological disorder not mentioned before he was recruited for the study and one patient turned out to have pilonidal sinus in combination with transsphincteric anal fistulation. Thus, $n=$ 102 patients who had planned excision of a pilonidal sinus were included and randomised to primary wound closure with a Limberg flap $(n=51)$ or to excision only with secondary wound healing $(n=51)$. Five patients were lost to follow-up (follow-up $95 \%$ ); we lost touch with four patients and one patient died. The number of patients that could be analysed was $n=48$ in the group with the Limberg flap procedure and $n=49$ in the group with excision only. An overview of the study methodology is shown in the CONSORT diagram in Fig. 2.

Both groups are comparable regarding baseline data as demonstrated in Table 1. In the group of patients with a 
Fig. 2 CONSORT diagram of the study

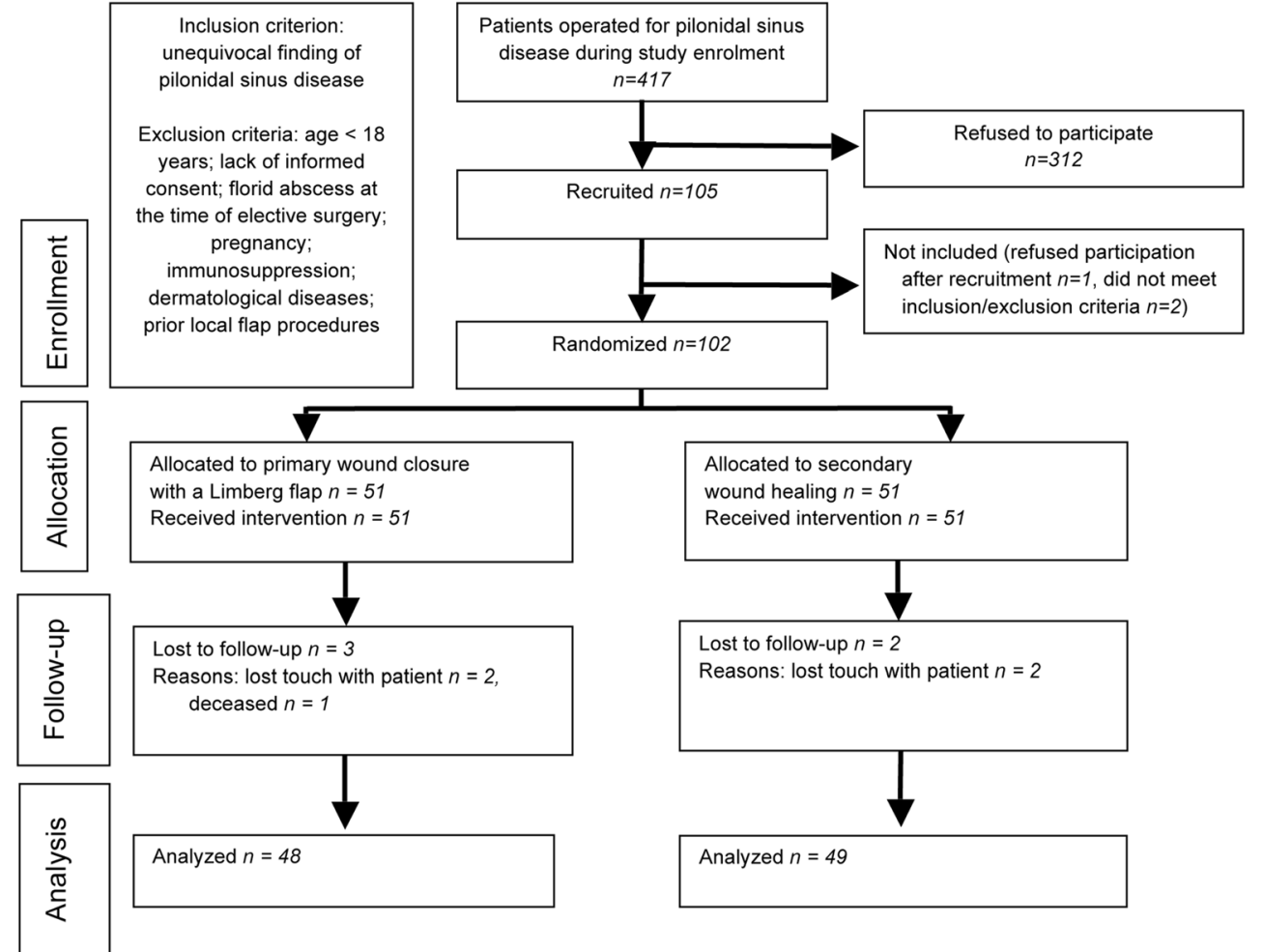

Limberg flap, intraoperative wound drainage was performed in $49 \%$ and wound tension was present in $10 \%$. The significant difference $(p<0.001)$ in operation time between the two groups is shown in Fig. 3.

The histological examination showed the presence of an abscess in $43 \%$, the presence of a granulocytic infiltrate in $54 \%$ and inclusion of hair in the sinus in $59 \%$ of the patients. Pilonidal sinus disease could be histologically confirmed in $96 \%$ of the patients.

The short-term results (at discharge and at 3 weeks postoperative) are shown in Table 2. The overall complication rates at 1 year postoperative were $49 \%$ in the patients with a Limberg flap vs. $12 \%$ in the patients with excision only $(p<0.001)$. The specific complications are shown in Table 3.
At 1-year follow-up, there was no significant difference between the two groups in mean patient satisfaction (9.2 in the group of patients with a Limberg flap vs. 8.3 in the group of patients with secondary wound healing, $p=0.97$ ) and in duration of incapacity for work $(p=0.52)$ as shown in the KaplanMeier curves in Fig. 4.

\section{Discussion}

We aimed to compare primary wound closure with a Limberg flap to secondary wound healing after excision of a pilonidal sinus. In contrast to the literature $[2,14]$, the results of this multicentre randomised controlled study show no advantage
Table 1 Baseline data of the randomised patients

\footnotetext{
a Two-sided Fisher's exact test

${ }^{\mathrm{b}}$ Wilcoxon rank-sum test
}

\begin{tabular}{llll}
\hline & $\begin{array}{l}\text { Limberg flap } \\
(n=51)\end{array}$ & $\begin{array}{l}\text { Excision only } \\
(n=51)\end{array}$ & $p$ value \\
\hline Median age (range) & $26(18-25)$ years & $24(18-52)$ years & $0.14^{\mathrm{b}}$ \\
Male gender & $84 \%(n=43)$ & $78 \%(n=40)$ & $0.61^{\mathrm{a}}$ \\
Incision prior to operation & $51 \%(n=26)$ & $57 \%(n=29)$ & $0.69^{\mathrm{a}}$ \\
$\begin{array}{l}\text { Median interval between incision and definitive } \\
\quad \text { surgical treatment }\end{array}$ & 13 days & 13 days & $0.71^{\mathrm{b}}$ \\
$\begin{array}{l}\text { Recurrent disease } \\
\text { Pores not located in the midline }\end{array}$ & $8 \%(n=4)$ & $18 \%(n=9)$ & $0.24^{\mathrm{a}}$ \\
$\begin{array}{l}\text { Median distance between pores and anus (range) } \\
\text { Median wound volume (range) }\end{array}$ & $51 \%(n=21)$ & $27 \%(n=14)$ & $0.21^{\mathrm{a}}$ \\
Median number of pores (range) & $30(5-16) \mathrm{cm}$ & $6(3-9) \mathrm{cm}$ & $0.65^{\mathrm{b}}$ \\
& $1(0-3+)$ & $30(3-150) \mathrm{ml}$ & $0.83^{\mathrm{b}}$ \\
\end{tabular}




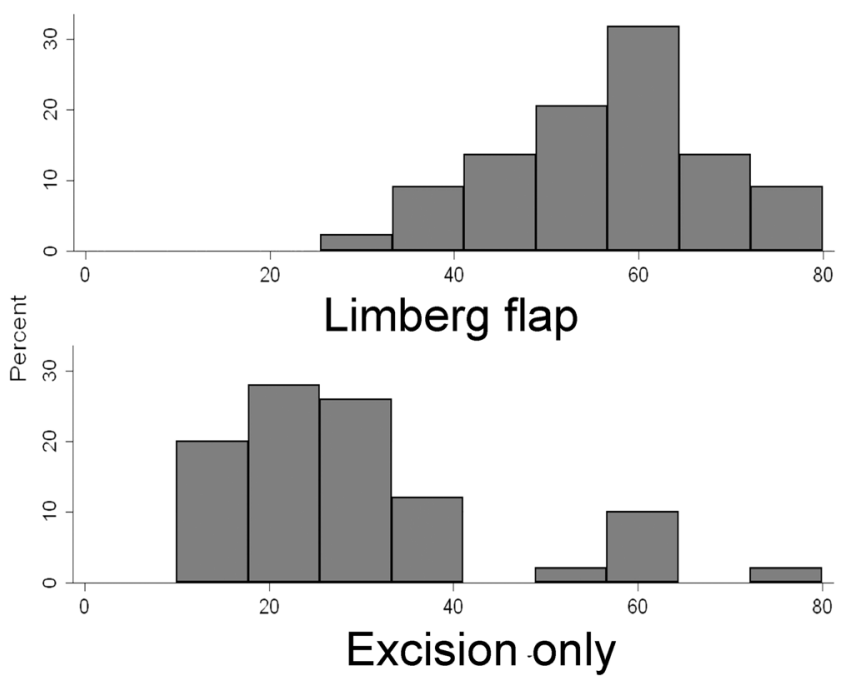

Fig. 3 Difference in operation time

of the Limberg flap procedure if compared to secondary wound healing. This is a relevant finding because the socioeconomic burden of pilonidal sinus disease is high and the optimal surgical therapy is still under debate.

The main reason for the lack of advantage of the Limberg flap procedure compared to excision only seems to be the rather high complication rate in this study compared to the literature $[23,24]$. Apart from postoperative complications, there certainly are several external factors influencing the incapacity for work, such as economic and psychological factors. However, as this study is a randomised controlled study, it is unlikely that these factors are not evenly distributed in the two groups.

As a known disadvantage of the Limberg flap technique, the duration of operation was significantly longer in the group of patients with the Limberg flap procedure compared to the group of patients with excision only [14]. Most operations were taught to residents by consultant surgeons; this explains the rather long duration of the operations in both groups.

This study did not aim to assess the long-term complication rates of the different surgical techniques, as it is known that recurrences after surgery for pilonidal sinus disease occur up to 20 years after surgery [21]. This is why we decided to do the latest follow-up by phone call at 1 year after surgery (followup rate $95 \%$ ). It could be argued that a follow-up by phone call might miss or overdiagnose some recurrences. However, the defined criteria for recurrence used in this study have also been defined as hard criteria for recurrence by other study groups for phone call follow-up; thus, we feel that our 1-year follow-up by phone call is reliable [25].

The complication rate was significantly higher in the group of patients with the Limberg flap procedure, compared to the group with excision only. The only postoperative wound complication in the group with excision only was wound healing disorder necessitating re-intervention. Such a delay in healing is known to occur due to infection and/or wound shapes hindering good drainage [26]. However, in this study, no routine examinations were done to exclude infection. Minor complications such as wound bleeding after surgery with intervention at the bedside were not assessed in this study.

The complications in the group with the Limberg flap procedure mainly were wound dehiscence and skin necrosis. It has to be underlined that the follow-up was done by thorough clinical examination by attending surgeons, and the majority of wound dehiscences were small and at the tip of the Limberg flap. To avoid this well-known complication, some use a modified Limberg flap technique placing the lower pole $1-2 \mathrm{~cm}$ lateral to the midline $[12,27]$, while others recommend tailoring around edge at the tip of the Limberg flap, as done by some surgeons in this study [28].

Another technical issue is if the Limberg flap has to include the fascia of the gluteus muscle [16] or not [28]. As mentioned in the "Patients and methods" section, we did not include the fascia of the gluteus muscle. Some wound dehiscences of the Limberg flap could have probably been avoided by modifying the operation technique.

There was no case of flap necrosis, and the skin necrosis reported was always at the cutting edge. Both complications probably reflect too much wound tension [29]. Additional complications in the group with the Limberg flap procedure were hematoma, seroma and probably consecutive infection [29].

Table 2 Results at discharge from hospital and at 3 weeks postoperative follow-up

\begin{tabular}{|c|c|c|c|}
\hline & Limberg flap $(n=48)$ & Excision only $(n=49)$ & $p$ value \\
\hline Mean (SD) pain score at discharge (graduated scale from 0 to 10 ) & $2.4(1.7)$ & $2.5(1.7)$ & $0.80^{\mathrm{b}}$ \\
\hline Intake of more than one pain killer at discharge & $35 \%(n=17)$ & $31 \%(n=15)$ & $0.67^{\mathrm{a}}$ \\
\hline Mean (SD) pain score at 3 weeks postop (graduated scale from 0 to 10 ) & $2.1(2.3)$ & $1.9(2.5)$ & $0.54^{\mathrm{b}}$ \\
\hline Mean (SD) satisfaction score at 3 weeks postop (graduated scale from 0 to 10 ) & $8.5(1.7)$ & $8.7(2.0)$ & $0.37^{\mathrm{b}}$ \\
\hline Percentage of patients at work at 3 weeks postop & $31 \%(n=15)$ & $22 \%(n=11)$ & $0.37^{\mathrm{a}}$ \\
\hline
\end{tabular}

$S D$ standard deviation

${ }^{\text {a }}$ Two-sided Fisher's exact test

${ }^{\mathrm{b}}$ Wilcoxon rank-sum test 
Table 3 Summarised postoperative complications at 1-year follow-up (one patient could have more than one complication, overall complication rate see Fig. 4)

${ }^{\text {a }}$ Two-sided Fisher's exact test

\begin{tabular}{llll}
\hline & $\begin{array}{l}\text { Limberg flap } \\
(n=48)\end{array}$ & $\begin{array}{l}\text { Excision only } \\
(n=49)\end{array}$ & $p$ value \\
\hline Seroma & $6 \%(n=3)$ & - & - \\
Wound dehiscence & $46 \%(n=22)$ & - & - \\
Skin necrosis & $10 \%(n=5)$ & $0 \%(n=0)$ & $0.027^{\mathrm{a}}$ \\
Hematoma & $6 \%(n=3)$ & - & - \\
Infection & $4 \%(n=2)$ & $0 \%(n=0)$ & $0.24^{\mathrm{a}}$ \\
Wound healing disorder necessitating re-intervention & - & $6 \%(n=3)$ & - \\
Recurrence rate at 1 year & $13 \%(n=6)$ & $6 \%(n=3)$ & $0.32^{\mathrm{a}}$ \\
\hline
\end{tabular}

There was no significant difference in recurrence rate between the two groups. As wound complications significantly influence the long-term recurrence rate [30], it could be anticipated that the long-term recurrence rate in the group with the Limberg flap procedure will be higher than that in the group with excision only.

Apparent abscesses were treated with incision and antibiotic therapy for median 13 days until the elective definitive surgical treatment was done. As patients, who were randomised to the excision only procedure, could have had their operation without delay, this could be a further argument for the excision only procedure.

\section{Limitations}

The main shortcoming of this study is the rather high number of patients that refused to participate in the study; thus, a certain degree of patient selection bias cannot be excluded. Indeed, a lot of these typically young patients refused to participate because they did not like to be allocated to a surgical technique by randomisation. Furthermore, the cosmetic result was an issue, and after being informed adequately about the pros and cons of the different techniques, a lot of patients preferred to choose the surgical technique on their own.

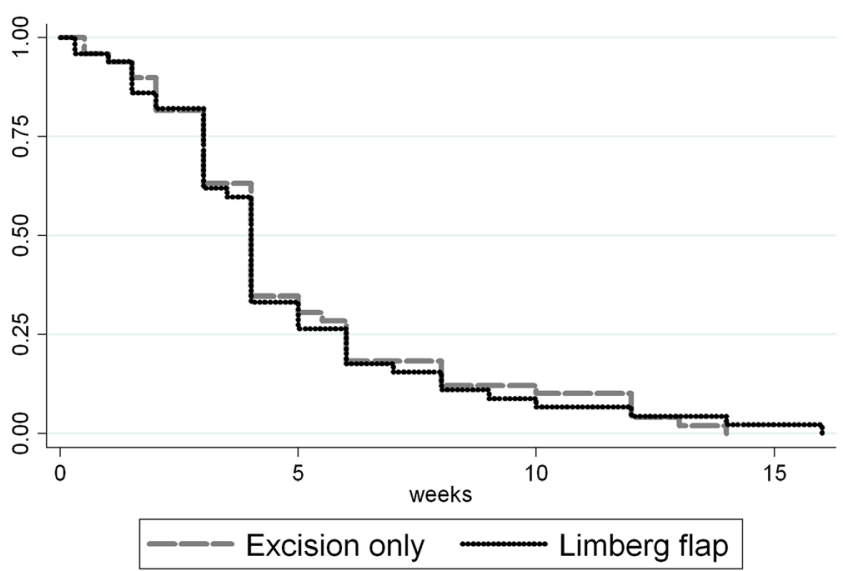

Fig. 4 Kaplan-Meier curve of incapacity for work
Although a high number of patients refused to participate in this study, the number of dropouts is very low (3\%) because randomisation was done just prior to surgery. This supports the validity of the results of this multicentre randomised controlled study.

\section{Conclusions}

After excision of a pilonidal sinus, primary wound closure with a Limberg flap has no advantage over secondary wound healing. The main reason for this conclusion is the relatively high complication rate of primary wound closure with a Limberg flap.

\section{References}

1. Evers T, Doll D, Matevossian E, Noe S, Neumann K, Li HL, Huser N, Ludde R, Hoffmann S, Krapohl BD (2011) Trends in incidence and long-term recurrence rate of pilonidal sinus disease and analysis of associated influencing factors. Zhonghua Wai Ke Za Zhi [Chin J Surg] 49(9):799-803

2. Al-Khamis A, McCallum I, King PM, Bruce J (2010) Healing by primary versus secondary intention after surgical treatment for pilonidal sinus. Cochrane Database Syst Rev 1, CD006213. doi:10.1002/ 14651858.CD006213.pub3

3. Loganathan A, Arsalani Zadeh R, Hartley J (2012) Pilonidal disease: time to reevaluate a common pain in the rear! Dis Colon Rectum 55(4):491-493. doi:10.1097/DCR.0b013e31823fee6c

4. Akinci OF, Bozer M, Uzunkoy A, Duzgun SA, Coskun A (1999) Incidence and aetiological factors in pilonidal sinus among Turkish soldiers. Eur J Surg=Acta Chir 165(4):339-342. doi:10.1080/ 110241599750006875

5. Clothier PR, Haywood IR (1984) The natural history of the post anal (pilonidal) sinus. Ann R Coll Surg Engl 66(3):201203

6. Doll D, Matevossian E, Hoenemann C, Hoffmann S (2013) Incision and drainage preceding definite surgery achieves lower 20-year longterm recurrence rate in 583 primary pilonidal sinus surgery patients. $\mathrm{J}$ Dtsch Dermatol Ges=J Ger Soc Dermatol: JDDG 11(1):60-64. doi: 10.1111/j.1610-0387.2012.08007.x 
7. Shabbir J, Chaudhary BN, Britton DC (2011) Management of sacrococcygeal pilonidal sinus disease: a snapshot of current practice. Int J Color Dis 26(12):1619-1620. doi:10.1007/s00384-011-1169-9

8. Limberg AA (1946) Mathematical principles of local plastic procedures on the surface of the human body. Medgis, Leningrad

9. Green AR (1986) Use of the rhomboid flap in pilonidal sinus. Ann R Coll Surg Engl 68(5):293-294

10. Karydakis GE (1973) New approach to the problem of pilonidal sinus. Lancet 2(7843):1414-1415

11. Horwood J, Hanratty D, Chandran P, Billings P (2012) Primary closure or rhomboid excision and Limberg flap for the management of primary sacrococcygeal pilonidal disease? A meta-analysis of randomized controlled trials. Color Dis: Off J Assoc Coloproctology G B Irel 14(2):143-151. doi:10.1111/j.1463-1318.2010.02473.x

12. Can MF, Sevinc MM, Hancerliogullari O, Yilmaz M, Yagci G (2010) Multicenter prospective randomized trial comparing modified Limberg flap transposition and Karydakis flap reconstruction in patients with sacrococcygeal pilonidal disease. Am J Surg 200(3): 318-327. doi:10.1016/j.amjsurg.2009.08.042

13. Bessa SS (2013) Comparison of short-term results between the modified Karydakis flap and the modified Limberg flap in the management of pilonidal sinus disease: a randomized controlled study. Dis Colon Rectum 56(4):491-498. doi:10.1097/DCR. 0b013e31828006f7

14. Jamal A, Shamim M, Hashmi F, Qureshi MI (2009) Open excision with secondary healing versus rhomboid excision with Limberg transposition flap in the management of sacrococcygeal pilonidal disease. JPMA J Pak Med Assoc 59(3):157-160

15. Karakayali F, Karagulle E, Karabulut Z, Oksuz E, Moray G, Haberal M (2009) Unroofing and marsupialization vs. rhomboid excision and Limberg flap in pilonidal disease: a prospective, randomized, clinical trial. Dis Colon Rectum 52(3):496-502. doi:10.1007/DCR. 0b013e31819a3ec0

16. Cihan A, Mentes BB, Tatlicioglu E, Ozmen S, Leventoglu S, Ucan BH (2004) Modified Limberg flap reconstruction compares favourably with primary repair for pilonidal sinus surgery. ANZ J Surg 74(4):238-242. doi:10.1111/j.1445-2197.2004.02951.x

17. Aydede H, Erhan Y, Sakarya A, Kumkumoglu Y (2001) Comparison of three methods in surgical treatment of pilonidal disease. ANZ J Surg 71(6):362-364

18. Sondenaa K, Nesvik I, Gullaksen FP, Furnes A, Harbo SO, Weyessa S, Soreide JA (1995) The role of cefoxitin prophylaxis in chronic pilonidal sinus treated with excision and primary suture. J Am Coll Surg 180(2):157-160

19. Milone M, Musella M, Salvatore G, Leongito M, Milone F (2011) Effectiveness of a drain in surgical treatment of sacrococcygeal pilonidal disease. Results of a randomized and controlled clinical trial on 803 consecutive patients. Int J Color Dis 26(12):1601-1607. doi:10.1007/s00384-011-1242-4

20. Katsoulis IE, Hibberts F, Carapeti EA (2006) Outcome of treatment of primary and recurrent pilonidal sinuses with the Limberg flap. Surgeon: J R Coll Surg Edinb Irel 4(1):7-10, 62

21. Sievert H, Evers T, Matevossian E, Hoenemann C, Hoffmann S, Doll D (2013) The influence of lifestyle (smoking and body mass index) on wound healing and long-term recurrence rate in 534 primary pilonidal sinus patients. Int J Color Dis. doi:10.1007/s00384-013-1731-8

22. Schulz KF, Altman DG, Moher D, Group C (2010) CONSORT 2010 statement: updated guidelines for reporting parallel group randomised trials. BMJ 340:c332. doi:10.1136/bmj.c332

23. Urhan MK, Kucukel F, Topgul K, Ozer I, Sari S (2002) Rhomboid excision and Limberg flap for managing pilonidal sinus: results of 102 cases. Dis Colon Rectum 45(5):656-659

24. Aslam MN, Shoaib S, Choudhry AM (2009) Use of Limberg flap for pilonidal sinus - a viable option. J Ayub Med Coll, Abbottabad: JAMC 21(4):31-33

25. Doll D, Krueger CM, Schrank S, Dettmann H, Petersen S, Duesel W (2007) Timeline of recurrence after primary and secondary pilonidal sinus surgery. Dis Colon Rectum 50(11):1928-1934. doi:10.1007/ s10350-007-9031-4

26. Marks J, Harding KG, Hughes LE, Ribeiro CD (1985) Pilonidal sinus excision-healing by open granulation. Br J Surg 72(8):637-640

27. Mentes BB, Leventoglu S, Cihan A, Tatlicioglu E, Akin M, Oguz M (2004) Modified Limberg transposition flap for sacrococcygeal pilonidal sinus. Surg Today 34(5):419-423. doi:10.1007/s00595-003-2725-x

28. Hegele A, Strombach FJ, Schonbach F (2003) Reconstructive surgical therapy of infected pilonidal sinus. Der Chirurg 74(8):749-52

29. McGregor ADMI (2000) Fundamental techniques of plastic surgery and their surgical applications, Tenthth edn. Churchill Livingstone, Edinburgh

30. Sondenaa K, Diab R, Nesvik I, Gullaksen FP, Kristiansen RM, Saebo A, Komer H (2002) Influence of failure of primary wound healing on subsequent recurrence of pilonidal sinus. Combined prospective study and randomised controlled trial. Eur J Surg=Acta Chir 168(11):614-618 